\title{
Prognostic value of 2-[18 F]fluoro-2-deoxy-D-glucose uptake as measured by PET scan in patients with non-small cell lung cancer
}

\author{
XIANG CHEN $^{1}$, JUN ZHAO $^{2}$, YI-HUI GUAN ${ }^{2}$, SHUN LU $^{3}$, CHUAN-TAO ZUO $^{2}$, \\ FENG-CHUN HUA ${ }^{2}$ and XIANGTONG LIN ${ }^{2}$
}

\author{
${ }^{1}$ Department of Nuclear Medicine, Shanghai First People's Hospital, Shanghai Jiaotong University, \\ 200080 Shanghai; ${ }^{2}$ Department of Nuclear Medicine and PET Center, Huashan Hospital, Fudan University, \\ 200235 Shanghai; ${ }^{3}$ Department of Lung Tumor Clinic Medicine Center, Chest Hospital, \\ Shanghai Jiaotong University, 200030 Shanghai, P.R. China
}

Received June 30, 2008; Accepted September 4, 2008

DOI: $10.3892 / \mathrm{mmr}_{-} 00000046$

\begin{abstract}
This study aimed to evaluate the prognostic value of 2-[ $\left.{ }^{18} \mathrm{~F}\right]$ fluoro-2-deoxy-D-glucose $\left({ }^{18} \mathrm{~F}-\mathrm{FDG}\right)$ uptake determined by positron emission tomography (PET) in patients with nonsmall cell lung cancer (NSCLC) in relation to disease stage and/or tumor histology. A retrospective review of 144 patients with newly diagnosed lung cancer undergoing PET imaging was performed. Differences in survival were compared by univariate and multivariate analyses. Univariate analysis identified three prognostic factors: stage, lesion size and the standardized ${ }^{18} \mathrm{~F}$-FDG uptake value. The latter was a better prognostic predictor in lung cancer patients with early-stage disease than in those at advanced stages. Multivariate analysis revealed that the most important prognostic factors were tumor-node-metastasis (TNM) stage and the standardized ${ }^{18} \mathrm{~F}-\mathrm{FDG}$ uptake value. Patients with standardized uptake values (SUVs) $>8$ had a 2.5 times higher mortality rate than those with values $\leq 8$. A one-unit increase in SUV corresponded with a $7 \%$ increase in the hazard of death. SUVs provided stronger prognostic stratification in patients with adenocarcinoma than in those with squamous cell carcinoma (SCC). Furthermore, the best choice of prognostic predictor differed between the two types of lung cancer: the SUV was best for SCC, while TNM stage was most significant for adenocarcinoma. In conclusion, ${ }^{18} \mathrm{~F}-\mathrm{FDG}$ uptake in primary lung lesions is an independent prognostic predictor in patients
\end{abstract}

Correspondence to: Dr Jun Zhao, Department of Nuclear Medicine and PET Center, Huashan Hospital, Fudan University, East Wuzhong Road 518, 200235 Shanghai, P.R. China

E-mail: huashan80@126.com

Key words: non-small cell lung cancer, positron emission tomography, X-ray computed, ${ }^{18} \mathrm{~F}$-fluorodeoxyglucose, standardized uptake value, prognosis with NSCLC, especially those with adenocarcinoma or earlystage disease. Further stratification of patients with the same TNM stage based on SUVs may allow for the modification of individual treatment strategies, resulting in improved outcome.

\section{Introduction}

Lung cancer is one of the leading causes of cancer-related death worldwide, with non-small cell lung cancer (NSCLC) being the main type. Accurate staging of NSCLC plays an important role in stratifying patients for optimal treatment regimens and improving prognosis. $2-\left[{ }^{18} \mathrm{~F}\right]$ fluoro-2-deoxy-Dglucose $\left({ }^{18} \mathrm{~F}-\mathrm{FDG}\right)$ positron emission tomography (PET) and PET/computerized tomography (CT) are non-invasive imaging techniques for the evalation of unidentified pulmonary nodules, the diagnosis of lung cancer, the staging of mediastinal lymph nodes and the evaluation of distant metastases and response to therapy $(1,2)$. In many countries, FDG PET is now included in the standard staging procedures for lung cancer, and its prognostic value is being evaluated. Previous studies have shown that FDG PET may provide prognostic information (3-13). One of the most frequently used criteria is the standardized uptake value (SUV) of FDG. While some studies show a significant correlation between SUV and survival, it is unclear whether SUV can act as an independent prognostic factor (14). The objective of this study was to determine whether SUV is an independent prognostic predictor in NSCLC patients and, if so, whether it has different prognostic values in patients at different stages of the disease or with different histology.

\section{Materials and methods}

Patients. Patients with newly diagnosed NSCLC undergoing FDG PET imaging at the PET center of Huashan Hospital were considered eligible for participation in this study. Indications for PET imaging included strongly suspected lung cancer based on chest radiographs and CT or a pathologically proven new diagnosis of lung cancer. Patients were excluded if they had received chemotherapy or radiotherapy before PET imaging, 
or if they had an expected survival time of $<3$ months. A total of 156 NSCLC patients, 112 men and 44 women, with a mean age of 62 years (range 30-84) were enrolled.

Each patient was assigned a stage according to the revised international staging system of the UICC. Clinical staging methods included physical examination, thoracic CT, bone scan, head CT or magnetic resonance imaging, mediastinoscopy and PET. To simplify staging, patients were categorized into group I (stages I and II) or group II (stages III and IV).

PET imaging. PET imaging was performed with either a Siemens ECAT EXACT HR ${ }^{+}$PET or a Siemens Biograph Sensation 16 PET/CT (Siemens Medical Solutions, Knoxville, TN, USA). Patients fasted for at least $6 \mathrm{~h}$ prior to the PET scan and were dosed with 5.55-7.4 MBq/kg of ${ }^{18} \mathrm{~F}-\mathrm{FDG}$ intravenously, followed by PET $1 \mathrm{~h}$ after administration of FDG. The blood sugar level before FDG injection was $<7 \mathrm{mMol} / 1$ for each subject.

Imaging interpretation. Two experienced investigators independently evaluated the PET scan results. In the event of disagreement, a consensus was reached before the results were used for analysis. Focal intense uptake was considered a positive result. Low-grade uptake with diffused areas or no uptake was confirmed along with the information provided by the CT image. SUV was calculated automatically from the PET image by selecting the region of interest (ROI) and using the equation SUV = activity concentration within the tissue defined by ROI/(injected dose/body weight), which gives the relative metabolic activity of a given lesion. The maximum SUV (SUVmax) was defined as the maximum FDG uptake within the region of the primary tumor on the PET image, and the mean SUV (SUVmean) was defined as the mean concentration of FDG uptake in the ROI. The SUVmax, which was less variable than the SUVmean, was used throughout data analysis.

Data collection and statistical analysis. Parameters used to describe patients included age, gender, lesion size, tumornode-metastasis (TNM) stage, SUV, histology, survival status and survival time. Survival time was defined as the time between the date of PET imaging and the date the patients succumbed to the disease or the date of the last follow-up. Statistical analysis was performed with the SPSS11.5 statistical package. Categorical variables were male vs. female, group I vs. II and squamous cell carcinoma (SCC) vs. adenocarcinoma, while numeric variables were age $\leq 65$ vs. $>65$ and lesion size $\leq 3 \mathrm{~cm}$ vs. $>3 \mathrm{~cm}$. Since, based on previous reports, the cut-off points for the SUV varied considerably (3-5,7-12), we used a log-rank test to determine the SUV cut-off point with the most statistical significance.

The significance of the differences in SUV between the two groups was evaluated by a two-tailed Student's t-test. Survival probabilities for the patient subgroups as defined by the co-variables were calculated by the Kaplan-Meier method and compared using the log-rank test. Multivariate analysis was accomplished with the Cox proportional hazards model, with forward stepwise covariate entry (likelihood ratio method) and significance levels of 0.05 for entry and 0.1 for removal. Reported P-values were two-sided, and $\mathrm{P}<0.05$ was considered statistically significant.
Table I. Patient characteristics

\begin{tabular}{lcc}
\hline Variable & No. of patients & Deceased patients \\
\hline Gender & 105 & 49 \\
Male & 39 & 12 \\
Female & & \\
Age & 67 & 31 \\
$>65$ & 77 & 30 \\
$\leq 65$ & & \\
Tumor size (cm) & 81 & 42 \\
$>3$ & 63 & 19 \\
$\leq 3$ & & \\
TNM stage & 72 & 16 \\
I+II & 72 & 45 \\
III+IV & & \\
Histology & & 45 \\
Adenocarcinoma & 107 & 37 \\
SCC & 37 & \\
SUV & & \\
$>8$ & 62 & \\
$\leq 8$ & 82 & \\
\hline
\end{tabular}

\section{Results}

A total of 156 patients with NSCLC were enrolled in the present study, including 107 patients with adenocarcinoma, 37 with SCC, eight with bronchioalveolar carcinoma and four with adenosquamous carcinoma. Due to small sample size, the latter two groups were excluded from analysis. Patient numbers in stages I, II, III and IV were 53, 19, 32 and 40, respectively, resulting in an equal number of 72 patients in groups I and II. During the follow-up period, 61 patients succumbed to the disease. The median follow-up period for the remaining 83 patients was 24 months (range 14-65). Patient characteristics are summarized in Table I.

Standardized uptake value. The mean SUV of primary lesions in the 144 patients was $7.8 \pm 4.3$ (median 7.5), with a range of 1.0-23.8. Twelve patients had a SUV $<2.5$, and 10 of these had stage I disease. Patients with advanced disease (group II) had higher SUVs than those in early stages (group I) $(8.7 \pm 4.2$ vs. $6.7 \pm 4.2, \mathrm{P}<0.05)$. Lesions with larger tumor size $(>3 \mathrm{~cm})$ had higher SUVs than those with smaller tumor size $(9.2 \pm 4.2$ vs. $6.6 \pm 4.3, \mathrm{P}<0.05)$. No statistical differences were found for SUVs between genders or age groups. Of note is that patients with SCC had higher SUVs than those with adenocarcinoma $(10.2 \pm 5.4$ vs. $6.9 \pm 3.5, \mathrm{P}<0.05)$.

Optimal standardized uptake cut-off value based on univariate survival analysis. Based on the log-rank test, a cut-off SUV of eight was the best discriminative value for predicting prognosis in all patients, with a SUV of nine for patients with adenocarcinoma and of eight for patients with SCC (Table II). 
Table II. Relationship between SUV cut-off values and survival based on the log-rank test.

\begin{tabular}{|c|c|c|c|c|c|c|}
\hline \multirow[b]{2}{*}{ SUV cut-off } & \multicolumn{2}{|c|}{ All patients } & \multicolumn{2}{|c|}{ Adeno } & \multicolumn{2}{|c|}{ SCC } \\
\hline & $\chi^{2}$ & P-value & $\chi^{2}$ & $\mathrm{P}$-value & $\chi^{2}$ & P-value \\
\hline 5 & 11.85 & 0.0006 & 6.54 & 0.0106 & 6.13 & 0.0133 \\
\hline 6 & 10.28 & 0.0013 & 6.28 & 0.0122 & 4.38 & 0.0363 \\
\hline 7 & 9.19 & 0.0024 & 5.54 & 0.0185 & 4.38 & 0.0363 \\
\hline 8 & 18.89 & $<0.0001$ & 12.44 & 0.0004 & 7.88 & 0.0050 \\
\hline 9 & 17.78 & $<0.0001$ & 14.16 & 0.0002 & 5.20 & 0.0226 \\
\hline 10 & 15.76 & 0.0001 & 13.55 & 0.0002 & 2.99 & 0.0838 \\
\hline
\end{tabular}

SCC, patients with squamous cell carcinoma; Adeno, patients with adenocarcinoma.

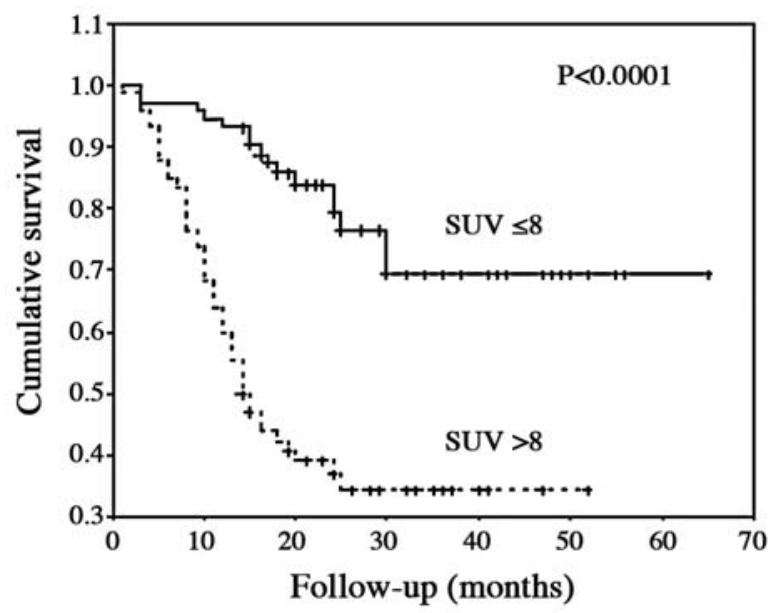

Figure 1. Survival curves among NSCLC cases stratified by the SUV of the ${ }^{18}$ F-FDG PET (Kaplan-Meier method).

Univariate analysis for all patients. In the present study, 62 patients had tumor lesions with a SUV $>8$ and a mean survival of 26.2 months [95\% confidence interval (CI) 20.9-31.5), while 82 had tumor lesions with a SUV $\leq 8$ and a mean survival of 47.9 months (95\% CI 42.2-53.7) (Fig. 1).

Survival was also affected by TNM stage and lesion size. Advanced stage and larger lesion size were correlated with poorer prognosis. The mean survival times of groups I and II were 51.2 months (95\% CI 45.2-57.2) and 25.3 months (95\% CI 20.6-30.1), respectively $(\mathrm{P}<0.0001)$. The mean survival times of patients with a lesion size $\leq 3 \mathrm{~cm}$ and $>3 \mathrm{~cm}$ were 47.4 months (95\% CI 40.8-54.0) and 29.6 months (95\% CI 24.9-34.3), respectively ( $\mathrm{P}=0.0064)$.

SUVs provided stronger prognostic stratification in group I than in group II ( $\mathrm{P}=0.0053$ vs. $\mathrm{P}=0.0094)$. Gender, age and tumor type had no predictive value in prognosis $(\mathrm{P}>0.05)$.

Univariate analysis for patients with adenocarcinoma or SCC. In patients with adenocarcinoma, the significant prognostic predictors were SUV, TNM stage and lesion size, with TNM stage being the most significant $(\mathrm{P}<0.0001)$. In patients with SCC, the significant predictors were SUV and TNM stage, with SUV being more significant than TNM stage. SUV was
Table III. COX proportional hazards model for 144 patients (using dichotomized variables).

\begin{tabular}{lrrr}
\hline Variable & P-value & HR & $95 \%$ CI HR \\
\hline Stage, group II vs. I & $<0.001$ & 3.732 & $2.090-6.663$ \\
Tumor SUV, >8 vs. $\leq 8$ & 0.001 & 2.504 & $1.483-4.226$ \\
\hline
\end{tabular}

a better predictor for patients with adenocarcinoma than for those with $\operatorname{SCC}(\mathrm{P}=0.0002$ vs. $\mathrm{P}=0.005)$.

Multivariate analysis for all patients. On the basis of the results of the univariate analyses, variables with the potential to be predictive factors were entered as candidate variables in the multivariate COX analysis. The variables for all patients and for those with adenocarcinoma included primary tumor size, SUV and stage. For those with SCC, the variables were SUV and stage.

Stage and SUV were significant prognostic factors, while lesion size had no statistical significance. Patients with advanced stages of disease or higher SUVs had poorer prognoses (Table III). The mortality of patients with a SUV $>8$ was 2.5 times higher than that of those with a SUV $\leq 8$. We incorporated the SUV as a continuous variable in the multivariate analysis and found it to be a significant predictor $(\mathrm{P}=0.008)$ with a hazard ratio (HR) of 1.07 (95\% CI 1.02-1.10), indicating that a one-unit increase in SUV corresponded with a $7 \%$ increase in the hazard of death.

Multivariate analysis for adenocarcinoma and SCC. In patients with adenocarcinoma, TNM stage and SUV affected survival, with stage being the better predictive factor $(\mathrm{P}<0.001$, $\mathrm{HR}=4.707,95 \%$ CI 2.232-9.925). In the case of SCC, SUV was the more significant prognostic factor $(\mathrm{P}=0.018, \mathrm{HR}=$ $11.708,95 \%$ CI $1.517-90.271)$.

\section{Discussion}

For NSCLC, the TNM staging system is an important tool used by clinicians to estimate prognosis and select the optimal 
treatment modalities. Since TNM staging does not consistently give a satisfactory explanation for differences in survival, it is important to identify other prognostic factors. The amount of FDG uptake can be presented by semi-quantitative measures such as SUV and compared between patients. Previous studies have correlated FDG uptake with lung tumor doubling time (15) and proliferation markers such as Ki67 (16). FDG uptake in the primary tumor correlates with poor survival (3-13). Most of these studies were based on comparisons of survival between groups of patients with high and low SUVs, defined by applying cut-offs for SUV ranging from 5 to 20 (3-5,7-12). For SUVs, there is apparently no true cut-off point, but rather a transition zone in which the prognosis gradually worsens. This is substantiated by the results of the present study, in which a range of SUVs from 5 to $10 \mathrm{had}$ discriminatory value, with the most discriminatory being 8 .

In a study of 2,700 lung cancer patients who underwent surgery, only $39 \%$ of the clinical staging results were consistent with pathological staging (17). Considering that PET scans are more sensitive and accurate than conventional staging methods such as CT (18), and that the post-PET stage provides a stronger prognostic stratification (6), in the present study we determined the TNM stage by combining a conventional staging system with PET findings.

In this study, univariate and multivariate analyses showed SUV to be an independent prognostic predictor for NSCLC patients. Cerfolio et al (11) reported that SUV is a better prognostic predictor than stage. However, our study and many others have shown that though SUV is a prognostic predictor independent of stage, TNM stage is the most valuable predictor of survival. We incorporated SUV as a continuous variable in multivariate analysis and observed a $7 \%$ increase in the hazard of death after a one-unit increase in SUV. This is in agreement with the results of Downey et al (9) and Borst et al (10), who observed a 7 and $6 \%$ increase in the hazard of death, respectively. Since patients of the same TNM stage may have a varying prognosis and recurrence, it is important for clinicians to subdivide these patients into different groups and to select different treatment strategies for them. The present study indicates that the determination of SUVs is an effective non-invasive method for subdividing patients, and is useful in developing strategies for individually adapted treatment. In brief, the SUV, in combination with conventional prognostic factors, may indicate whether a patient is an appropriate candidate for more aggressive treatment.

We also explored whether SUVs have different prognostic value for patients with different cancer stages or histology. SUVs provided stronger prognostic stratification in group I than in group II. The reason may be that large differences in survival were not expected in group II, since more than half of the patients had stage IV disease with a relatively low survival probability. We therefore speculate that SUV is a better predictor of survival or recurrence in early-stage lung cancer patients than in advanced-stage patients. A limitation of this study is that, since the patients were divided into only two groups according to stage, the prognostic value of SUVs for patients with different stages of cancer needs to be confirmed by further comparison.

Our study furthermore indicates that SUV may be a better prognostic predictor in adenocarcinoma than in SCC. The reason is possibly related to the higher expression of the glucose transporter (19) and, thus, to the relatively higher FDG uptake in SCC than in adenocarcinoma. Multivariate analysis demonstrated that the best choice of prognostic predictor differs between these two cell types of lung cancer, with SUV being the best predictor for SCC and TNM stage being the most significant factor (better than SUV) for adenocarcinoma. However, since the number of patients with SCC was relatively small, this result warrants further study.

In conclusion, ${ }^{18} \mathrm{~F}-\mathrm{FDG}$ uptake based on PET scan is an independent prognostic predictor in NSCLC patients, especially for those with adenocarcinoma or early-stage disease. These results may help physicians subdivide patients with the same TNM stage in order to develop strategies that optimize individual treatments and improve clinical outcome.

\section{References}

1. Bunyaviroch $\mathrm{T}$ and Coleman RE: PET evaluation of lung cancer. J Nucl Med 47: 451-469, 2006.

2. Bruzzi JF and Munden RF: PET/CT imaging of lung cancer. J Thorac Imaging 21: 123-136, 2006.

3. Ahuja V, Coleman RE, Herndon J and Patz EF Jr: The prognostic significance of fluorodeoxyglucose positron emission tomography imaging for patients with non-small cell lung carcinoma. Cancer 83: 918-924, 1998.

4. Vansteenkiste JF, Stroobants SG, De Leyn PR, Verbeken EK, Deneffe GJ, Mortelmans LA and Demedts MG: Prognostic importance of the standardized uptake value on ${ }^{18} \mathrm{~F}$-fluoro-2deoxy-glucose - positron emission tomography scan in nonsmall cell lung cancer: an analysis of 125 cases. J Clin Oncol 17: 3201-3206, 1999.

5. Dhital K, Saunders CA, Seed PT, O'Doherty MJ and Dussek J: ${ }^{18}$ F-Fluorodeoxyglucose positron emission tomography and its prognostic value in lung cancer. Eur J Cardiothorac Surg 18: 425-428, 2000.

6. Hicks RJ, Kalff V, MacManus MP, Ware RE, Hogg A, McKenzie AF, Matthews JP and Ball DL: ${ }^{18} \mathrm{~F}-\mathrm{FDG}$ PET provides high-impact and powerful prognostic stratification in staging newly diagnosed non-small cell lung cancer. J Nucl Med 42: 1596-1604, 2001.

7. Higashi K, Ueda Y, Arisaka Y, Sakuma T, Nambu Y, Oguchi M, Seki H, Tonami $\mathrm{H}$ and Yamamoto I: ${ }^{18} \mathrm{~F}-\mathrm{FDG}$ uptake as a biologic prognostic factor for recurrence in patients with surgically resected non-small cell lung cancer. J Nucl Med 43: 39-45, 2002.

8. Jeong HJ, Min JJ, Park JM, Chung JK, Kim BT, Jeong JM, Lee DS, Lee MC, Han SK and Shim YS: Determination of the prognostic value of ${ }^{18} \mathrm{~F}$-fluorodeoxyglucose uptake by using positron emission tomography in patients with non-small cell lung cancer. Nucl Med Commun 23: 865-870, 2002.

9. Downey RJ, Akhurst T, Gonen M, Vincent A, Bains MS, Larson S and Rusch V: Preoperative F-18 fluorodeoxyglucosepositron emission tomography maximal standardized uptake value predicts survival after lung cancer resection. J Clin Oncol 22: 3255-3260, 2004

10. Borst GR, Belderbos JS, Boellaard R, Comans EF, De-Jaeger K, Lammertsma AA and Lebesque JV: Standardised FDG uptake: a prognostic factor for inoperable non-small cell lung cancer. Eur J Cancer 41: 1533-1541, 2005.

11. Cerfolio RJ, Bryant AS, Ohja B and Bartolucci AA: The maximum standardized uptake values on positron emission tomography of a non-small cell lung cancer predict stage, recurrence, and survival. J Thorac Cardiovasc Surg 130: 151-159, 2005.

12. Eschmann SM, Friedel G, Paulsen F, Reimold M, Hehr T, Budach W, Scheiderbauer J, Machulla HJ, Dittmann H, Vonthein R and Bares R: Is standardised ${ }^{18}$ F-FDG uptake value an outcome predictor in patients with stage III non-small cell lung cancer? Eur J Nucl Med Mol Imaging 33: 263-269, 2006.

13. Kramer H, Post WJ, Pruim J and Groen HJM: The prognostic value of positron emission tomography in non-small cell lung cancer: analysis of 266 cases. Lung Cancer 52: 219-224, 2006.

14. Kieninger AN, Welsh R, Bendick PJ, Zelenock G and Chmielewski GW: Positron emission tomography as a prognostic tool for early-stage lung cancer. Am J Surg 191: 433-436, 2006. 
15. Duhaylongsod FG, Lowe VJ, Patz EF Jr, Vaughan AL, Coleman RE and Wolfe WG: Lung tumor growth correlates with glucose metabolism measured by fluoride-18 fluorodeoxyglucose positron emission tomography. Ann Thorac Surg 60: 1348-1352, 1995 .

16. Vesselle H, Schmidt RA, Pugsley JM, Li M, Kohlmyer SG, Vallieres E and Wood DE: Lung cancer proliferation correlates with [F-18]fluorodeoxyglucose uptake by positron emission tomography. Clin Cancer Res 6: 3837-3844, 2000.

17. Huang GJ, Zhang DC, Mao YS, Li J, Wang YG, Wang DL, Xue Q, et al: Comparison of clinical and surgico-pathological TNM stage of 2007 lung cancer patients. Chin J Oncol 27: 551-552, 2005.
18. Lardinois D, Weder W, Hany TF, Kamel EM, Korom S, Seifert B, von Schulthess GK and Steinert HC: Staging of nonsmall-cell lung cancer with integrated positron-emission tomography and computed tomography. N Engl J Med 348: 2500-2507, 2003.

19. Ito T, Noguchi Y, Satoh S, Hayashi H, Inayama Y and Kitamura H: Expression of facilitative glucose transporter isoforms in lung carcinomas: its relation to histologic type, differentiation grade, and tumor stage. Mod Pathol 11: 437-443, 1998. 